\title{
Editorial
}

\section{Biomedical Applications of Colloidal Nanocrystals}

\author{
Marek Osinski, ${ }^{1}$ Thomas M. Jovin, ${ }^{2}$ and K. Yamamoto ${ }^{3}$ \\ ${ }^{1}$ Center for High Technology Materials, University of New Mexico, 1313 Goddard SE, Albuquerque, NM 87106-4343, USA \\ ${ }^{2}$ Department of Molecular Biology, Max Planck Institute for Biophysical Chemistry, 37070 Göttingen, Germany \\ ${ }^{3}$ Research Institute, International Medical Center of Japan, 1-21-1 Toyama, Shinjuku-ku, Tokyo 162-8655, Japan \\ Correspondence should be addressed to K. Yamamoto, backen@ri.imcj.go.jp
}

Received 6 May 2008; Accepted 6 May 2008

Copyright (c) 2007 Marek Osinski et al. This is an open access article distributed under the Creative Commons Attribution License, which permits unrestricted use, distribution, and reproduction in any medium, provided the original work is properly cited.

Because of the high affinity of the thiol groups to a metal such as gold and zinc, we can conjugate metal nanocrystals with chemical compounds which contain thiol such as cysteine, cyctein contained peptide, and thiol-modified nucleic acids including DNA. Among them, T. Pellegrino et al. investigated Gold-DNA conjugates in detail by a comprehensive gelelectrophoresis study based on 1200 gels. The investigation contains the following three results: firstly, the precise detection of relative changes in size upon binding of molecules, secondary, comparison of the specific attachment of DNA via gold-thiol bonds to Au nanoparticles with nonspecific adsorption of DNA, and thirdly, determination of the maximum number of DNA molecules bounded per particle. These data might be the essential information for designing a functional nanodevice such as for the nanometer-sized electric circuit and for the carrier for gene delivery system.

A new ligand which readily binds itself to CdSe-ZnS coreshell QDs, via surface ligand exchange, was designed and synthesized by Kimihiro Susumu et al. from tetraethylene glycol (TEG) based bidentate ligand functionalized with dihydrolipoic acid (DHLA) and biotin (DHLA-TEG-biotin). Quantum dots capped with DHLA and DHLA-TEG-biotin or polyethylene glycol modified DHLA (DHLA-PEG600) are dispersed easily in aqueous buffer solutions. Homogenized buffer solutions of QDs capped with mixture of DHLAPEG600 and DHLA-TEG-biotin are stable over broad pH range and showed specific interactions with NeutrAvidin in surface binding assays. The authors say "further studies of these surface functionalized QDs for coupling with a variety of bioreceptors and biological assays are in progress."

For the study on the ligand-receptor binding, we need fluorescent markers. Ligand conjugated quantum dots are reported here by Ian D. Tomlinson et al. for imaging GABAc receptor on the surface membrane of intact cell. They have already succeeded in developing serotonin-conjugated quantum dots and the method to reduce nonspecific binding using PEGylated quantum dots. Together with all these works, they have proceeded for imaging GABAc receptors heterologously expressed on Xenopus laevis oocytes.

On the other hand, Dopamine conjugated quantum dots are known to be endocytozed by cells bearing dopamine receptors and not by cells without dopamine receptors. Rafael Khatchadourian et al. studied the tools of fluorescence intensity and intermittency for following dopamine bioconjugate processing in living cells.

In this special issue, we have three papers on quantum dots as for the use of Forster (or fluorescent) resonance energy transfer (FRET). The characters of quantum dots such as photostability, high quantum yield broad absorption, and narrow emission spectrum are more useful for probes than organic dyes, especially in the study of molecular imaging.

In the investigation of E. Z. Chong et al., biotinylated DY731-Bio fluorophores with an absorption peak at $720 \mathrm{~nm}$ were self-assembled onto Qdot705-STVs that emit the fluorophores at $705 \mathrm{~nm}$ using a streptavidin-biotin binding mechanism. They have particular interest on the far-red region to minimize optical region absorption within tissue and to avoid cell autofluorescence and they succeeded in doing so.

In order to identify selective inhibitors of protein kinases efficiently, Ibrahim Yildiz et al. designed a binding assay to probe the interactions of human phosphoinositidedependent protein kinase-1 with potential ligands using FRET between quantum dots and organic dyes.

Using terbium complexes as donors and quantum dots as acceptors, FRET immunoassay is improved more than two orders of magnitude compared to commercial systems, 
which has been investigated by Niko Hildebrandt et al. They say "the presented results demonstrate the great potential of Tb to QD-FRET system for highly sensitive homogenous immunoassays for biological as well as clinical and medical applications."

For the application of MRI imaging, the following two papers are included. Mi Kyong Yoo et al. developed superparamagnetic iron oxide nanoparticles (SPIONs). SPIONs were coated with agent (PVLA-coated SPIONs) to be recognized by asialoglycoprotein receptors on hepatocytes. Intracellular uptake of this nanoparticle was visualized by confocal laser scanning microscopy and the hepatocytespecific delivery was also investigated with magnetic resonance image of rat liver. The authors said "the results suggest the potential utility of PVLA-coated SPIONs as livertargeting MRI contrast agent."

Quantum dots are absolutely useful for clinical laboratory test. Dilan Qin et al. say "tuberculosis is a global health threatening emergency with the spread of acquired immunodeficiency syndrome and the emergence of a drugresistant strain of mycobacterium tuberculosis." They used RuBpy-doped silica nanoparticles as the fluorescent probe to detect the bacteria with an indirect immunofluorescence microscopy. Four-hour assay time is enough including spiked sputum sample pretreatment, which can be done by the advantage of higher luminescence and higher photostability for the nanoparticles.

For the study on the clinical treatment, we have a paper of Magdalena M. Stevanović et al. They produced copolymer poly(D,L-lactide-co-glycolide) (DLPLG) nanoparticles by using physical method with solvent/nonsolvent systems and technique of the centrifugal processing. Encapsulation of ascorbic acids into DLPLG particles can be used for transdermal controlled delivery of ascorbic acids. The authors say "DLPLG nanospheres are very efficient means of transdermal transport of medicament within the polymer matrix."

Finally, this special issue includes two review articles. One is from Arthur M. Iga et al. They describe the clinical potential of quantum dots focused in sentinel lymph node mapping, cancer imaging, photodynamic therapy in cancer treatment, and drug delivery. The authors examined the hindrances for clinical use and the current progress in abrogating their inherent toxicity. The other review is from Camilla Luccardini et al. They start from the chemical and optical properties of nanocrystals and surface chemistry. They cross the description of plasma membrane section and reach the specific intracellular targets. They also describe a whole animal imaging in vivo with nanocrystals and cytotoxity of cadmium ion as an example.

Both of the authors of the review articles have taken the topics of toxicity commonly. Silica coating of the quantum dots might be one of the ways to escape from the cytotoxity. Masanori Ando et al. say "here we report the preparation of silica particle phosphor incorporating ZnSe-based NCs prepared by a reverse micelle method." The size distribution of the spherical particles is $20-40 \mathrm{~nm}$ in diameter. They have already reported the presentation of green- and red-emitting silica particles. "These emitting silica particles are expected to be applicable as fluorescent biomarkers," they also say. Their method of silica coating might be applicable for any type of the nanoparticles.

On the other hand, Arthur M. Iga et al. say "with improvements in nanotechnology, nanotoxicology, and chemistry some of the goals could be achieved, however, it will be difficult to replace the toxic core of the QD without losing optical properties or we will redirect our efforts to less toxic elements." All of us might have to think about it deeply.

Marek Osinski

Thomas M. Jovin

K. Yamamoto 

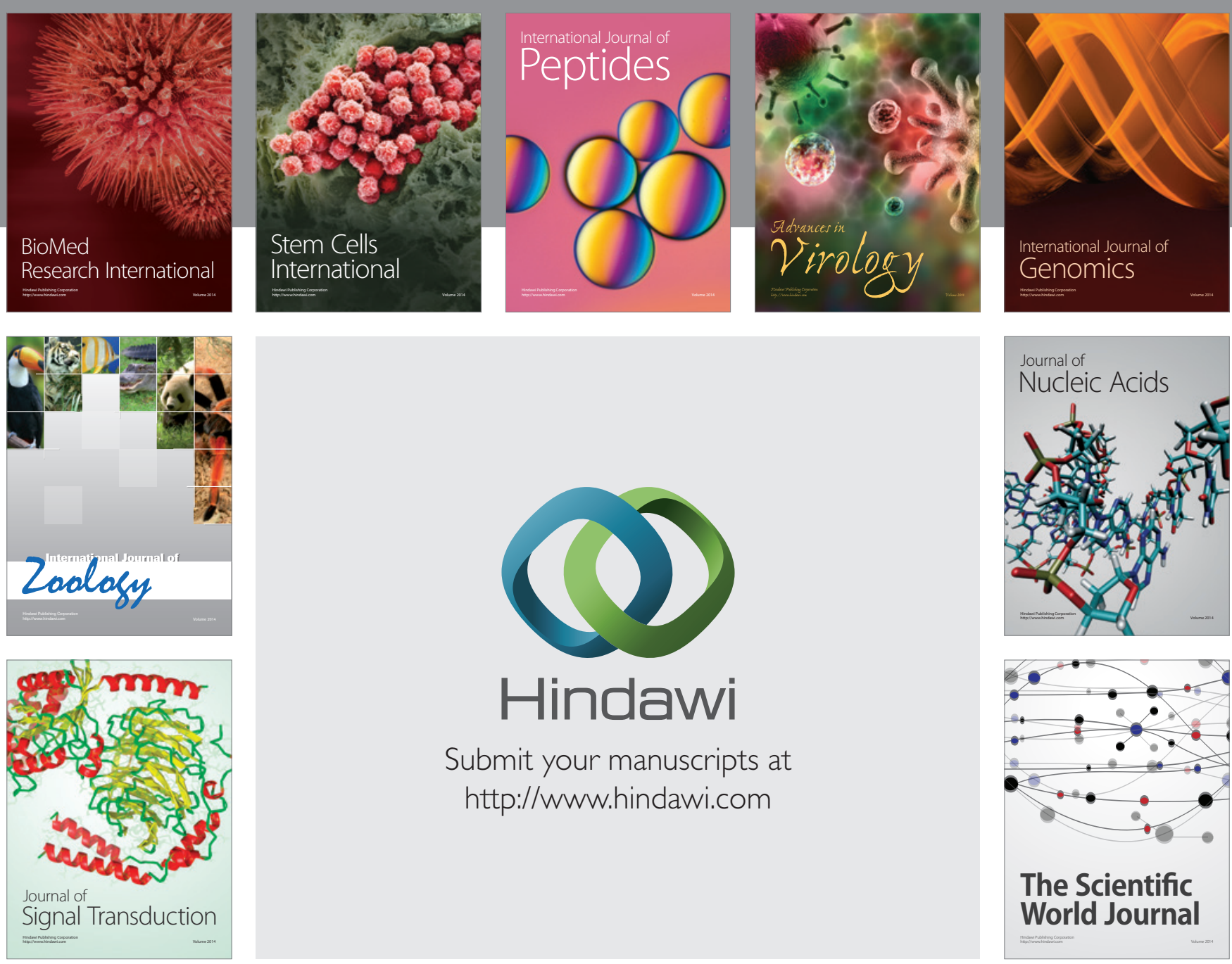

Submit your manuscripts at

http://www.hindawi.com
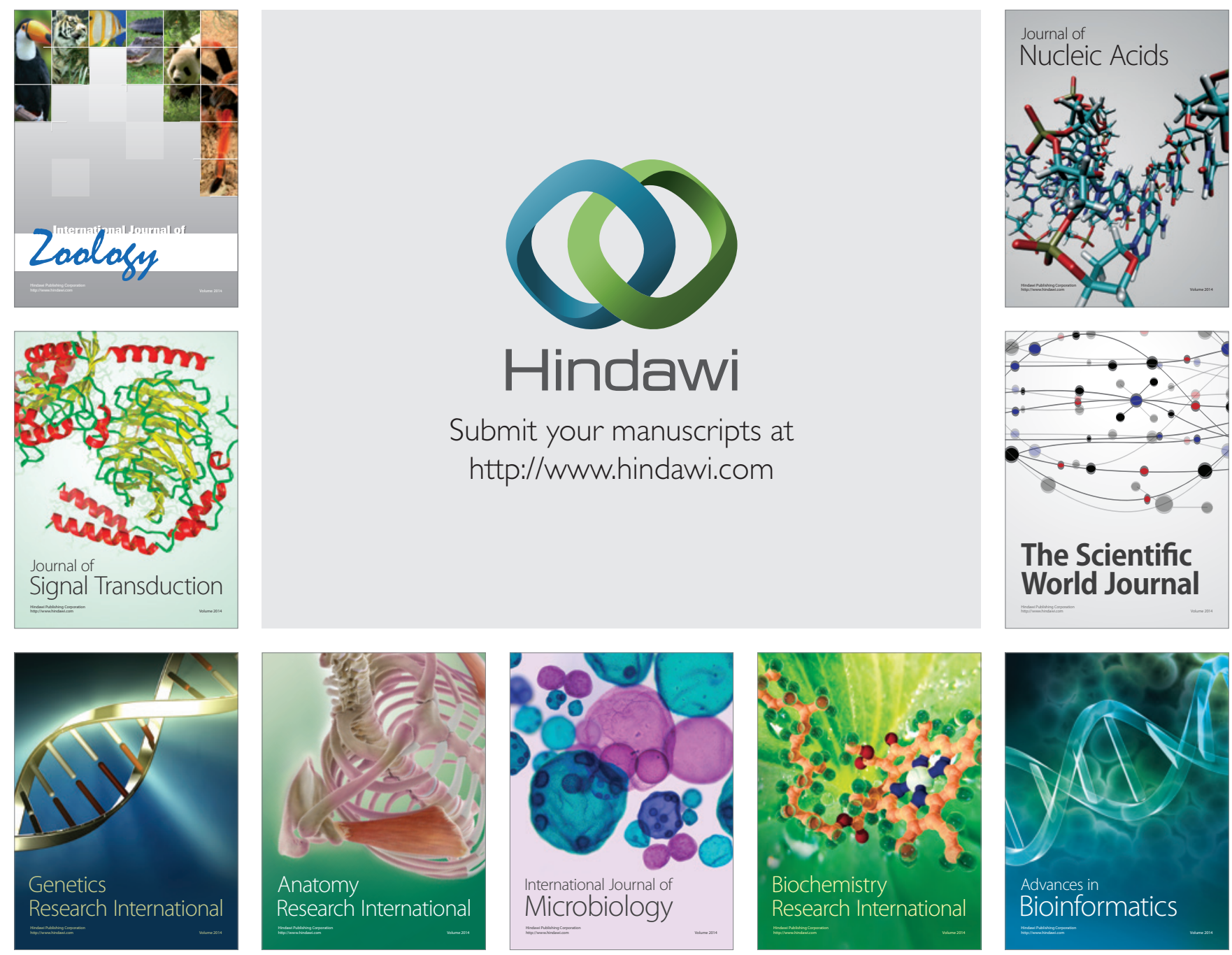

The Scientific World Journal
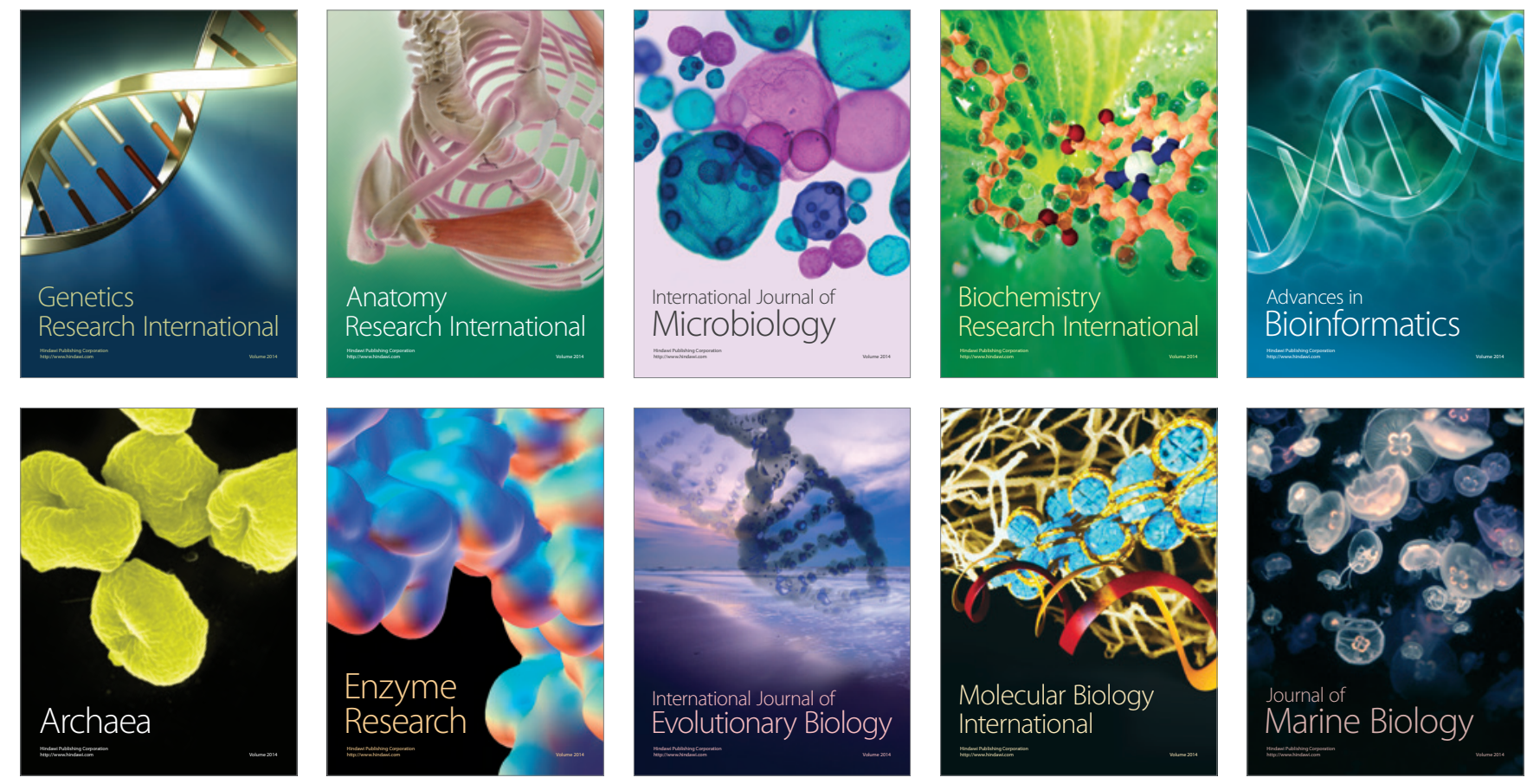